\title{
A novel biparametric magnetic resonance imaging sequence provides a more efficient and similarly efficacious method of risk stratifying men clinically suspected of having prostate cancer
}

\author{
Megan Stout, Tasha Posid, Shawn Dason \\ Department of Urology, The Ohio State University, Columbus, OH, USA \\ Correspondence to: Shawn Dason, MD, FRCSC. Assistant Professor of Urology, The Ohio State University, 915 Olentangy River Road, Suite 3100 , \\ Columbus, OH 43212, USA. Email: Shawn.Dason@osumc.edu. \\ Provenance: This is an invited article commissioned by the Section Editor Xiao Li (Department of Urology, Jiangsu Cancer Hospital \& Jiangsu \\ Institute of Cancer Research \& Nanjing Medical University Affiliated Cancer Hospital, Nanjing, China). \\ Comment on: Jambor I, Verho J, Ettala O, et al. Validation of IMPROD biparametric MRI in men with clinically suspected prostate cancer: a \\ prospective multi-institutional trial. PLoS Med 2019;16:e1002813.
}

Submitted Sep 09, 2019. Accepted for publication Sep 18, 2019.

doi: $10.21037 /$ tau.2019.09.35

View this article at: http://dx.doi.org/10.21037/tau.2019.09.35

Magnetic resonance imaging (MRI) for men suspected of having prostate cancer has become the standard of care. This is supported by the publication of high-quality data that it reduces the need for prostate biopsy and the diagnosis of clinically insignificant cancers, while increasing the diagnosis of clinically significant cancers (1-3).

The article "Validation of IMPROD biparametric MRI in men with clinically suspected prostate cancer: a prospective multi-institutional trial" (4) describes a large, prospective multicenter clinical trial that developed a novel biparametric MRI (bpMRI) protocol with the aim of decreasing imaging time (to 15 minutes), increasing cost effectiveness, and enhancing standardization. Those with a clinical suspicion of prostate cancer underwent bpMRI with all patients then undergoing systematic TRUS biopsy. Those with a suspicious lesion on bpMRI (Likert score 3-5) also had targeted biopsy either by cognitive technique or UroNav (Invivo, Gainesville, FL, USA) fusion. The study demonstrated bpMRI had a high accuracy $(70 \%)$ and negative predictive value (95\%) for clinically significant prostate cancer (Gleason Grade $\geq 3+4$ ). If only those with a suspicious bpMRI were biopsied, there would be a $22 \%$ reduction in the number of men undergoing biopsy.

This bpMRI technique addresses some limitations of MRI that have hampered widespread adoption. These barriers include the prolonged acquisition time of images and resultant high cost. bpMRI accomplishes this by reducing acquisition time to $15 \mathrm{~min}$ and eliminating the use of endorectal coils and IV contrast agents. This novel bpMRI technique has been studied in Finland. It is welldocumented that MRI scans in the United States cost more than MRI scans conducted internationally. It will be especially interesting to see an analysis of how this technique might reduce costs outside the Finnish setting, namely in the United States with our higher MRI costs.

Another strength of this study is that bpMRI performed similarly to other studies that have assessed the utility of multiparametric (mpMRI) for biopsy. The $4 M$ (5) and MRI-FIRST (3) studies both showed a similar clinically significant cancer rate detected by targeted and systematic biopsy. bpMRI showed a $46 \%$ rate with targeted biopsy and $49 \%$ rate with systematic biopsy in those with MRI lesions (Likert 3-5). The addition of targeted biopsy diagnosed an additional 13 clinically significant cases in the study cohort. This translates into an increase in clinically significant prostate cancer of $4.9 \%$ if those with MRI lesions receive both a targeted and systematic biopsy, a figure that is comparable to both 4M (5) and MRI-FIRST (3).

The main limitations of this study are reproducibility. The IMPROD trial utilized a Likert scoring system: a fivetiered scale describing the likelihood of prostate cancer in MRI from 1 being highly unlikely to 5 being very likely that clinically significant prostate cancer is present. Local radiologists in the study, as well as a designated central 
reader, had experience with the Likert scoring system, and each image's score stratified which individuals would eventually receive targeted plus systemic or systemic prostate biopsy alone for further evaluation (4). This is significantly different from the standardized PI-RADS v2.1 system used in clinical practice $(6,7)$. The aim of the PIRADS v2.1 system is to improve detection, localization, characterization and risk stratification of prostate cancer through accepted and standardized parameters established for mpMRI. It is uncertain how the bpMRI technique will be reproduced in other centers, especially when it does not use PIRADS v2.1.

MRI has clear utility in prostate cancer diagnosis and academic efforts need to focus on optimization and translation into clinical practice. Despite concerns surrounding the reproducibility of bpMRI, this study should be commended for finding that bpMRI has comparable results to studies on mpMRI, while improving upon some barriers that limit MRI utilization. bpMRI shows promise and further efforts will need to focus on how this technique can be used outside of Finland without the use of PIRADS v2.1. Cost analyses and comparison to mpMRI will be critical prior to widespread adoption.

\section{Acknowledgments}

None.

\section{Footnote}

Conflicts of Interest: The authors have no conflicts of interest to declare.

Ethical Statement: The authors are accountable for all aspects of the work in ensuring that questions related to the accuracy or integrity of any part of the work are appropriately investigated and resolved.

\section{References}

1. Ahmed HU, El-Shater Bosaily A, Brown LC, et al. Diagnostic accuracy of multi-parametric MRI and TRUS biopsy in prostate cancer (PROMIS): a paired validating confirmatory study. Lancet 2017;389:815-22.

2. Kasivisvanathan V, Rannikko AS, Borghi M, et al. MRItargeted or standard biopsy for prostate-cancer diagnosis. N Engl J Med 2018;378:1767-77.

3. Rouvière $\mathrm{O}$, Puech $\mathrm{P}$, Renard-Penna $\mathrm{R}$, et al. Use of prostate systematic and targeted biopsy on the basis of multiparametric MRI in biopsy-naïve patients (MRIFIRST): a prospective, multicenter, paired diagnostic study. Lancet Oncol 2019;20:100-9.

4. Jambor I, Verho J, Ettala O, et al. Validation of IMPROD biparametric MRI in men with clinically suspected prostate cancer: a prospective multi-institutional trial. PLoS Med 2019;16:e1002813.

5. van der Leest M, Cornel E, Israël B, et al. Head-to-head comparison of transrectal ultrasound-guided prostate resonance imaging with subsequent magnetic resonanceguided biopsy in biopsy-naïve men with elevated prostatespecific antigen: A large prospective multicenter clinical study. Eur Urol 2019;75:570-8.

6. Turkbey B, Rosenkrantz AB, Haider MA, et al. Prostate imaging reporting and data system version 2.1: 2019 update of prostate imaging reporting and data system version 2. Eur Urol 2019;76:340-51.

7. American College of Radiology: Prostate Imaging \& Reporting Data System (PI-RADS). Available online: https://www.acr.org/Clinical-Resources/Reporting-andData-Systems/PI-RADS
Cite this article as: Stout M, Posid T, Dason S. A novel biparametric magnetic resonance imaging sequence provides a more efficient and similarly efficacious method of risk stratifying men clinically suspected of having prostate cancer. Transl Androl Urol 2019;8(Suppl 5):S484-S485. doi: 10.21037/ tau.2019.09.35 